\title{
Comparison of time-motion analysis of conventional stool culture and the BD MAX'M Enteric Bacterial Panel (EBP)
}

\author{
Joel E. Mortensen ${ }^{1 *}$, Cindi Ventrola ${ }^{1}$, Sarah Hanna ${ }^{1}$ and Adam Walter ${ }^{2}$
}

\begin{abstract}
Background: Conventional bacterial stool culture is one of the more time-consuming tests in a routine clinical microbiology laboratory. In addition, less than $5 \%$ of stool cultures yield positive results. A molecular platform, the BD MAX' ${ }^{\text {TM }}$ System (BD Diagnostics, Sparks, MD) offers the potential for significantly more rapid results and less hands-on time. Time-motion analysis of the BD MAX Enteric Bacterial Panel (EBP) (BD Diagnostics, Quebec, Canada) on the BD MAX System was compared to conventional stool culture in the microbiology laboratory of a tertiary care pediatric hospital.

Methods: The process impact analysis of time-motion studies of conventional cultures were compared to those of EBP with 86 stool specimens. Sample flow, hands-on time, processing steps, and overall turnaround time were determined and analyzed. Data were obtained and analyzed from both standard operating procedures and direct observation. A regression analysis was performed to ensure consistency of measurements. Time and process measurements started when the specimens were logged into the accessioning area of the microbiology laboratory and were completed when actionable results were generated.
\end{abstract}

Results: With conventional culture, negative culture results were available from 41:14:27 (hours:minutes:seconds) to 54:17:19; with EBP, positive and negative results were available from 2:28:40 to 3:33:39.

Conclusions: This study supports the suggestion that use of the EBP to detect commonly encountered stool pathogens can result in significant time savings and a shorter time-to-result for patients with acute bacterial diarrhea.

Keywords: Time-motion analysis, BD MAX ${ }^{\mathrm{TM}}$, Diarrhea, Bacterial stool pathogens

\section{Background}

The World Health Organization has reported that, worldwide, there are nearly 1.7 billion cases of diarrheal disease every year and that diarrheal disease is the second leading cause of death in children under five years old [1,2]. Each year, diarrhea results in approximately 760,000 preventable deaths of children under the age of five years. Diarrhea in this age group is also a leading cause of malnutrition. Most cases of this disease are related to unsafe drinking-water, inadequate sanitation, and poor hygiene $[1,2]$.

Detection and identification of the etiological agents of acute bacterial diarrhea are important for both the

\footnotetext{
* Correspondence: joel.mortensen@cchmc.org

'Department of Laboratory Medicine, Cincinnati Children's Hospital,

MLC1010, 3333 Burnet Ave, 45229 Cincinnati, OH, USA

Full list of author information is available at the end of the article
}

treatment of individual patients and for the management of diarrheal diseases of public health importance. Conventional bacterial culture remains the gold standard for the aforementioned detection, even though stool culture has relatively low sensitivity and requires a significant amount of labor. The use of nucleic acid amplification methods to detect and identify the etiological agents of acute bacterial diarrhea could have a significant impact on the laboratory diagnostic process, clinical approach, and epidemiology of this disease [3-5].

The objective of this study was to examine the laboratory impact of a new molecular platform (use of the BD MAX Enteric Bacterial Panel on the BD MAX System) on turnaround time, associated laboratory processes, and the cost of providing results with this system compared to conventional culture methods. Results of both 
conventional culture (including a commercial immunoassay for shiga-toxin) and the EBP, which include tests for the detection of Salmonella spp., Shigella spp./ Enteroinvasive Escherichia coli (EIEC), Campylobacter spp. (jejuni and coli), and Shiga toxin 1 and 2 genes in stool specimens were evaluated. Lean and Six Sigma processes were used to analyze the time from sample receipt to actionable result for conventional stool culture and the EBP. The following "events or decisions per specimen" were determined: any action or thought process that must occur to process and issue a result, the overall distance traveled per sample as a measure of efficiency, and the operating costs of the two systems [6].

(The results of this study were presented, in part, at the 24th European Congress of Clinical Microbiology and Infectious Diseases, Barcelona, Spain, May 10-13, 2014 and at the 114th General Meeting of the American Society for Microbiology, Boston MA, May 17-20, 2014.)

\section{Methods}

Lean and Six Sigma processing analysis were performed to evaluate time-to-results for both culture and EBP testing. By design, this study did not involve human subjects or any patient information. Observations were performed without patient identifiers and additional testing was carried out on discarded, anonymous samples. Any sample ordered for routine stool culture was eligible for inclusion in the study.

\section{Culture}

Clinical stool samples were immediately accessioned and plated upon arrival in the laboratory following standard laboratory practices. They were not stored prior to culture. Sample flow, hands-on time, processing steps, overall turnaround time, and specimen travel distance were measured by two independent observers over the course of three separate observation periods; each observation period was five days. To eliminate any potential of operator-to-operator bias during the study, 11 different laboratory technologists were observed performing all pre-analytical, analytical, and post-analytical culture procedures which occurred at five different laboratory stations: specimen receipt, specimen plating and incubation, culture reading, automated identification (Vitek 2 System, bioMérieux, Marcy l'Etoile, France) and shigatoxin testing (Immunocard STAT! EHEC, Meridian Bioscience, Cincinnati, Ohio, USA).

In brief, for this study, stool samples that were submitted for routine culture were transported in Cary Blair Transport Medium (Meridian Bioscience, Inc.). Specimens were processed within $2 \mathrm{~h}$ of receipt. Initially, samples were inoculated onto the following agar media: $5 \%$ sheep blood, MacConkey, Sorbitol MacConkey, Hektoen, Campy CVA (BD Diagnostics, Sparks, MD, USA).
Cultures were incubated under standard conditions. Suspected bacterial pathogens were identified using the Vitek 2 System (bioMerieux) and standard conventional methodologies as needed. Additional testing may have included the following: Salmonella serotyping and Shigella serotyping Becton, Dickinson and Company, Sparks, MD, USA) and Remel RIM E. coli O157:H7 Latex Test (Remel, Lenexa, KS, USA).

Additional data were obtained and analyzed from laboratory Standard Operating Procedures (SOP) used routinely in this particular laboratory. In order to ensure consistency during the study, a regression analysis of measurements, processing, and adherence to the SOP was performed. Correlation studies were performed on independent data sets to ensure no bias was falsely introduced by operator-to-operator performance [7]. The following elements were analyzed: elapsed time, distance traveled, processing steps performed, and clinical decisions, from the time the specimens were logged into the accessioning area until the time actionable results were generated. Processing was observed and data were collected during each of the following notable events: specimen arrival, specimen accessioning, specimen plating and preparation, specimen incubation, first-day plate reading and workup, subsequent day(s) reading, including Campylobacter spp. cultures reading, automated identification and additional workup, shiga-toxin broth inoculation, shiga-toxin rapid testing, and verification of results, and entering results into the laboratory/hospital information system.

\section{EBP testing}

Methods similar to those used to evaluate culture processes were measured and analyzed: elapsed time, distance traveled, processing steps, and clinical decisions (also from the time specimens were logged into the accessioning area to the time actionable results were generated). Processing was observed and data were collected during each of the following notable events: specimen arrival, specimen accessioning, control preparation, specimen preparation, instrument preparation, worklist preparation, instrument processing, and result verification. For BD MAX testing, the samples were batched and tested in different batch sizes. Batch sizes ranged from 4 to 24 samples in increments of 4 to mimic routine clinical testing.

\section{Cost analysis}

Standard institutional cost analysis was used to determine the costs for conventional culture and for EBP. The main cost components of the analysis were labor, direct materials and supplies, and general shared costs (Test Site Burden). Hands-on time (minutes) for each step of the cultures and the EBP was multiplied by the 
average hourly technologist salary/min of labor to determine labor costs. The quantity of each item and the cost of each item used in culture and EBP testing were derived from institutional inventory data. Institutional overhead or Test Site Burden is the cost for basic services such as lights and heat and the cost of common shared laboratory equipment such as incubators, repeat pipettes, etc.

\section{Institutional review board}

It was determined that this study did not meet regulatory criteria for research involving human subjects because the research did not obtain data through intervention or interaction with the individual or identifiable private information. All observations of process were made without any patient identifiers available to the observer. All specimens tested on the BD MAX were anonymous, discarded samples that were only used after clinical testing was completed and for which no patient identifiers were used.

\section{Results}

86 patient specimens were examined. No pathogens under consideration in this study (i.e., Salmonella, Campylobacter, Shigella and shiga toxin producing organisms) were detected by culture or BD MAX EBP.

To enable a comparison, simultaneously, 84 alternate specimens tested by EBP were processed across six batches of differing size (4 to 24 samples each) on the BD MAX platform. Processing and turnaround times of routine cultures were compared to the process and turnaround times of EBP testing. The mean time to reportable result for 86 routine cultures was 44:37:00 (hours:minutes:seconds) (+/- $8 \mathrm{~h}, 10 \mathrm{~min}$ ) (Fig. 1). If potential pathogens were detected that required additional testing, the time to final result ranged from 97:18:17 to 145:27:11.

Although the time to perform EBP testing is approximately two hours, EBP is designed and best used to batch specimen testing at reasonable intervals as determined by each laboratory. If a reasonable operational model is two EBP runs per day (one in the morning, one in the afternoon), the time to reportable EBP results would be, at most, 07:06:00 (no standard deviation; all variability dependent upon batch size and timing of run). Hands-on time per specimen was 0:01:30 (+/19 s). With an assumption of two EBP runs per day and $90 \mathrm{~s}$ hands-on time/specimen, there was an $85 \%$ reduction of time to reportable results compared to culture.

\section{Process Steps for Culture and EBP}

Technologists made an average of 141 and 25 decisions per culture and EBP test, respectively. Thus, EBP testing required $82 \%$ fewer decisions than did culture (Table 1 ). The number of steps and processes in each unique laboratory can be represented by a spaghetti diagram of process flow for culture and for EBP testing (Fig. 2).

\section{Cost analysis}

Detailed costs are listed in Table 2. The basic labor to process and handle a stool culture was 0:15:00 - 0:17:00. Approximately $20 \%$ of the cultures required additional process steps to rule out potential pathogens; these additional steps resulted in additional labor (0:35:00 0:40:00) and supplies. The EBP required 0:01:28 hands on time.

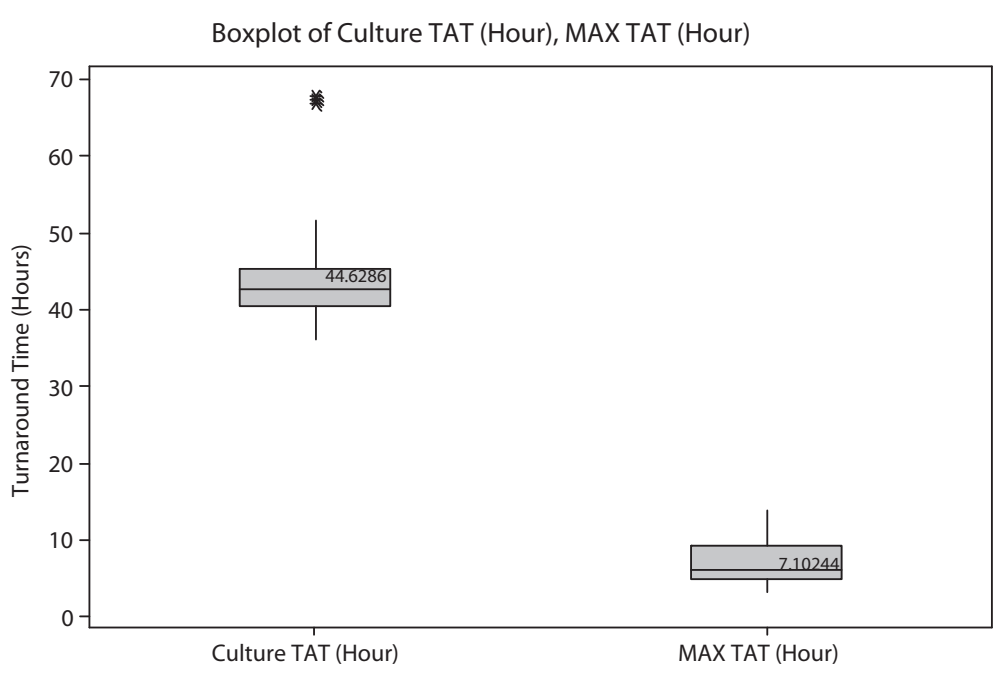

Fig. 1 The mean turnaround time (TAT) to reportable results for 86 routine stool cultures and 84 samples tested with BD MAX EBP. Legend. *Represents 4 outlying culture results that required additional testing for confirmation of the results. All final culture results were negative for pathogens 
Table 1 Average Number of Process Steps (Decisions/ Manipulations) Involved in Routine Culture and BD MAX EBP Testing

\begin{tabular}{lll}
\hline Process Steps & Routine Culture & EBP \\
\hline Receipt & 3 & 3 \\
Accession & 7 & 1 \\
Routine culture & 43 & - \\
Blood agar & 26 & - \\
MacConkey agar & 26 & - \\
Hektoen & 14 & - \\
Sorbitol MacConkey & 4 & - \\
Shiga toxin testing & - & 8 \\
Sample Preparation & - & 13 \\
System operation & 141 & 25 \\
Total activities &
\end{tabular}

\section{Discussion}

A number of molecular platforms have been evaluated for specific specimen types, including stool, and potential pathogens in clinical laboratory settings and have been shown to be highly sensitive and specific when compared to conventional methods [3-5]. The BD MAX platform has been evaluated for the detection of MRSA and more recently stool pathogens $[8,9]$. Recently, several investigators have recognized that beyond scientific validation, these platforms need to be evaluated for their impact on the operations and the time to reportable results in clinical laboratories [7, 10-12].

One of the more challenging parts of this study was accounting for all of the costs. The lack of positive samples with target stool pathogens did not allow a complete determination of the costs and labor associated with routine stool cultures. A community outbreak of acute bacterial diarrhea might significantly impact both labor and supplies for both of the methods in this study. In addition, it was difficult to account for the individual variability between technologists in the workup of stool cultures. Differences in individual technologists and their experience could have affected the extent of work and supplies needed for a culture. Nonetheless, including multiple technologists in the performance of this study more accurately represents real-world performance of the two methods than, for example, performing the study with specified research technologists. The use of a significant amount of shared equipment for routine cultures makes complete accounting for the portion of the cost of equipment such as water baths, incubators, storage rack, microscopes, etc. assigned to each culture difficult. At our institution, we use the somewhat arbitrary "Test Site Burden" as one mechanism of sharing these

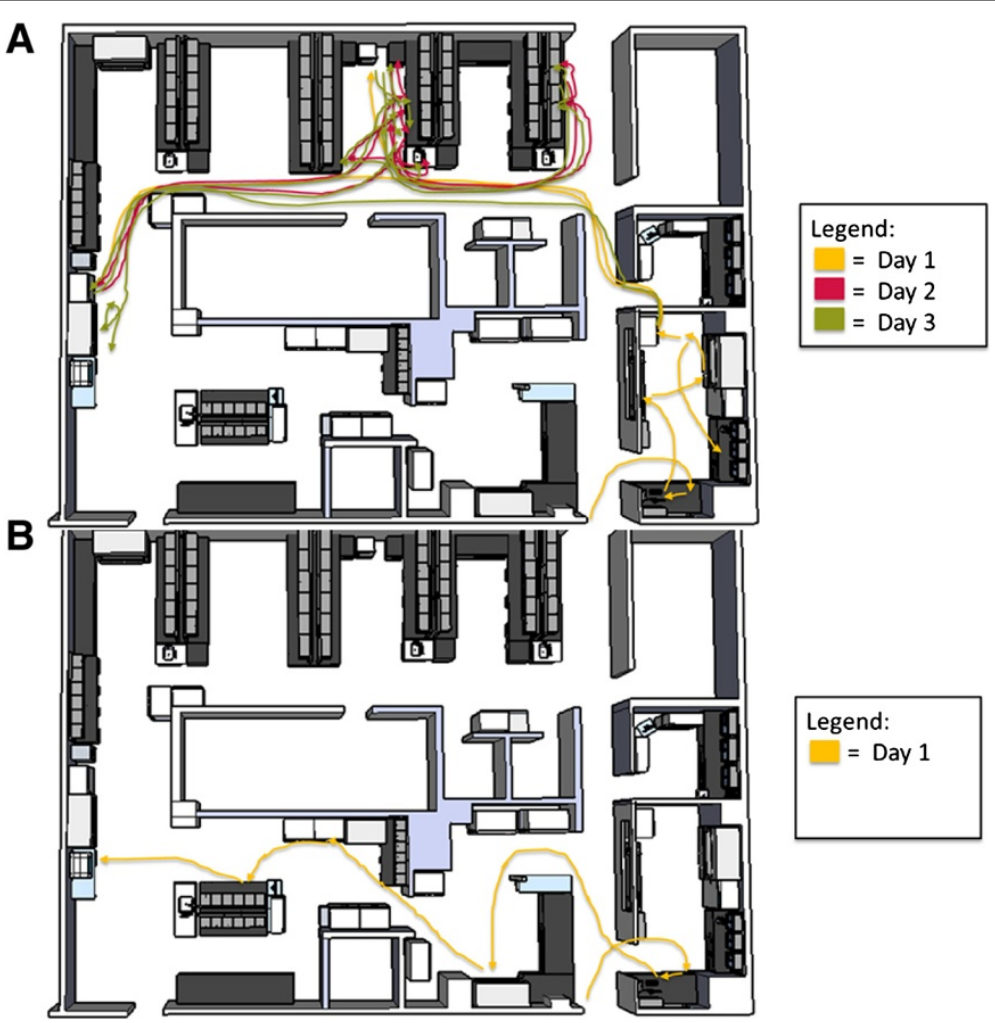

Fig. 2 Spaghetti Diagram of Process Flow for Routine Stool Culture (a) and BD MAX EBP Testing (b) 
Table 2 Cost Analysis of Routine Stool Cultures and BD MAX EBP Testing

\begin{tabular}{|c|c|c|c|c|c|c|}
\hline & \multicolumn{3}{|c|}{ Stool Culture } & \multicolumn{3}{|l|}{ EBP } \\
\hline & Cost/Unit & \#Units & Cost & Cost/Unit & \#Units & Cost \\
\hline \multicolumn{7}{|l|}{ Basic test } \\
\hline Labor -Technologist time (minutes) & 0.45 & $15-17$ & $6.75-7.65$ & 0.45 & 1.48 & 0.67 \\
\hline Information System labels & 0.05 & 3 & 0.15 & 0.05 & 2 & 0.1 \\
\hline 5 \% Sheep Blood agar plate & 0.25 & 1 & 0.25 & & & \\
\hline MacConkey agar plate & 0.25 & 1 & 0.25 & & & \\
\hline Campy agar plate and BioBag & 2.39 & 1 & 2.39 & & & \\
\hline Hektoen Enteric agar plate & 0.31 & 1 & 0.31 & & & \\
\hline MacConkey Sorbitol agar plate & 0.44 & 1 & 0.44 & & & \\
\hline Shiga toxin test kit and MacConkey Broth & 13.52 & 1 & 13.52 & & & \\
\hline Disposable $10 \mu$ l loop & & & & 0.02 & 1 & 0.02 \\
\hline Enteric Panel Kit & & & & $30-35.00$ & 1 & $30-35.00$ \\
\hline MAX test cartridge & & & & $0.40-0.65$ & 1 & $0.40-0.65$ \\
\hline Test site burden & 1.00 & 2 & 2.00 & 1.00 & 1 & 1.00 \\
\hline \multicolumn{7}{|l|}{ Additional workup* } \\
\hline Labor -Technologist time (minutes) & 0.45 & $35-40$ & $15.75-18.00$ & & & \\
\hline 5 \% Sheep Blood agar plate & 0.25 & $1-3$ & $0.25-0.75$ & & & \\
\hline Vitek-Gram negative ID card & 6.00 & $1-3$ & $6.00-18.00$ & & & \\
\hline RIM EC 0157:H7 test & 0.59 & 1 & 0.59 & & & \\
\hline Total Cost (in \$) & & & $\$ 26.06-64.30$ & & & $\$ 32.19-37.44$ \\
\hline
\end{tabular}

${ }^{*} 20 \%$ of cultures required additional labor and supplies to rule out potential pathogens

costs. An additional impact within the laboratory is the shift in supplies storage. A significant number of different media and tests are need for routine cultures and most of these require refrigerated storage. A move to the EBP assay would reduce the number of tests and the amount of media. In addition, adoption of the system would shift much of that storage to room temperature.

In contrast to culture, the cost of operating the $\mathrm{BD}$ MAX was more easily captured. However, there are several additional issues that affect the cost of operating the EBP assay that a clinical laboratory would need to consider. Because of how the disposables are constructed, samples can be run in various size batches. To optimize and reduce cost, the ideal batch size is 24 . To optimize and reduce turnaround time, the ideal batch size is as small as possible. Use of fewer stools samples per batch would have a minor effect on the cost of the test. The cost of the EBP would not change with a positive result; however, a positive EBP would require a follow-up culture to allow serotyping, antimicrobial susceptibility testing as appropriate, and epidemiological studies, including time and supplies to send isolates to the State Public Health Laboratory. Depreciation of instruments is an important consideration if the instrumentation is purchased outright. Our analysis did not include the cost of the analyzer as that cost per assay is directly related to the volume of assays performed on the instrument. Finally, the cost of service contracts is often not considered as part of the cost of a test, but can represent a significant cost to the operations of the laboratory.

As clinical microbiology laboratories move from traditional culture based methods to instrument based molecular methods, we need to look carefully at scientific merits of the various options, but we need also to look at the turnaround time of results, associated laboratory processes, and the cost of providing results with this system compared to conventional culture methods in our laboratories.

\section{Conclusion}

This study supports the suggestion that use of the BD MAX EBP can save significant time (over that required by culture) in the laboratory diagnosis of acute bacterial diarrhea caused by Salmonella spp., Shigella spp./(EIEC), Campylobacter spp. (jejuni and coli), and Shiga toxin producing $E$. coli which are responsible for $95 \%$ of acute bacterial gastroenteritis. The use of a flexible and focused approach to identifying enteric pathogens (bacteria, viruses \& parasites) based on patient history or risk, clinical presentation or clinician's preference is aligned with widely recommended clinical algorithms which not only potentially streamline laboratory testing and workflow in a cost effective manner, but also provide physicians with timely results which improve the 
standard of care for common causes of gastroenteritis. As additional nucleic amplification assays become available, the impact of the use of focused versus comprehensive panels will continue to be evaluated for their respective clinical relevance, cost and work flow implications.

\section{Abbreviations}

EBP: Enteric bacterial panel; EIEC: Enteroinvasive Escherichia coli; SOP: Standard operating procedures; MRSA: Methicillin resistant Staphylococcus aureus.

\section{Competing interests}

AW is an employee of BD Diagnostics. JEM has received honoraria from BD Diagnostics. Other authors have no competing interests.

\section{Authors' contributions}

JEM conceived the study, analyzed the data and authored the manuscript. CV performed data collection, participated in the process measurements and performed the cost analysis. SH performed the BD MAX testing and data collection, and participated in the process measurements. AW contributed to the study design and performed the process measurements. All authors have reviewed the manuscript and accept responsibility for its content.

\section{Acknowledgements}

BD Diagnostics (Sparks, MD) provided financial support and supplies for this project. BD Diagnostics did not design the study, interpret the data, write the manuscript or control submission of the manuscript for publication.

\section{Author details}

'Department of Laboratory Medicine, Cincinnati Children's Hospital, MLC1010, 3333 Burnet Ave, 45229 Cincinnati, OH, USA. ${ }^{2}$ BD Diagnostics, sparks, MD, USA.

Received: 8 December 2014 Accepted: 19 May 2015

Published online: 28 May 2015

\section{References}

1. WHO/UNICEF. Ending preventable child deaths from pneumonia and diarrhoea by 2025:The integrated Global Action Plan for Pneumonia and Diarrhoea (GAPPD). New York, N.Y: The United Nations CHildren's Fund(UNICEF)/World Health Organization (WHO); 2013.

2. Johansson EW, Wardlaw T, Binkin N, Brocklehurst C, Dooley T. Diarrhoea: why children are still dying and what can be done, (UNICEF). New York, N.Y: The United Nations CHildren's Fund(UNICEF)/World Health Organization (WHO); 2009.

3. Navidad JF, Griswold DJ, Gradus MS, Bhattacharyya S. Evaluation of Luminex xTAG gastrointestinal pathogen analyte-specific reagents for high-throughput, simultaneous detection of bacteria, viruses, and parasites of clinical and public health importance. J Clin Microbiol. 2013;51:3018-24.

4. Buchan BW, Olson WJ, Pezewski M, Marcon MJ, Novicki T, Uphoff TS, et al. Clinical evaluation of a real-time PCR assay for identification of Salmonella, Shigella, Campylobacter (Campylobacter jejuni and C. coli), and shiga toxin-producing Escherichia coli isolates in stool specimens. J Clin Microbiol. 2013;51(12):4001-7.

5. Patel A, Navidad J, Bhattacharyya S. Site-specific Clinical Evaluation of the Luminex xTAG Gastrointestinal Pathogen Panel for the Detection of Infectious Gastroenteritis in Fecal Specimens. J Clin Microbiol. 2013;52(8):3068-71.

6. Schweikhart SA, Dembe AE. The application of Lean and Six Sigma techniques to clinical and translational research. J Invest Med. 2009;57(7):748-55.

7. Felder RA, Foster ML, Lizzi MJ, Pohl BR, Diemert DM, Towns BG. Process evaluation of a fully automated molecular diagnostics system. J Assoc Lab Autom. 2009;14:262-8.

8. Widen R, Healer V, Silber S. Laboratory Evaluation of the BD MAX MRSA assay. J Clin Microbiol. 2014;52(7):2686-8.

9. Harrington SM, Buchan B, Doern C, Fader R, Ferraro MJ, Pillai D, et al. Multi-center evaluation of the BD MAX ${ }^{\mathrm{TM}}$ Enteric Bacterial Panel PCR assay for the rapid detection of Salmonella spp., Shigella spp., Campylobacter spp. (C. jejuni and C. coli), and Shiga toxin 1 and 2 genes. ASM General Meeting, 2014.
10. Hassell LA, Glass CF, Yip C, Eneff PA. The combined postive impact of Lean methology and Ventana Symphony autostainer on histology lab workflow. BMC Clin Path. 2010;10:2-10.

11. Felder RA, Jackson KD, Walter AM. Process Evaluation of an Open Architecture Real-Time Molecular Laboratory Platform. J Lab Autom. 2014;19(5):468-73.

12. Williams JA, Eddleman $L$, Pantone A, Martinez R, Young S, Van Der Pol B. Time-Motion Analysis of Four Automated Systems for the Detection of Chlamydia trachomatis and Neisseria gonorrhoeae by Nucleic Acid Amplification Testing. J Lab Autom. 2013;19(4):423-6.

\section{Submit your next manuscript to BioMed Central and take full advantage of:}

- Convenient online submission

- Thorough peer review

- No space constraints or color figure charges

- Immediate publication on acceptance

- Inclusion in PubMed, CAS, Scopus and Google Scholar

- Research which is freely available for redistribution

Submit your manuscript at www.biomedcentral.com/submit 